\title{
Best-shot Versus Weakest-link in Political Lobbying: An Application of Group All-pay Auction*
}

\author{
Subhasish M. Chowdhury ${ }^{\mathrm{a}}$ and Iryna Topolyan ${ }^{\mathrm{b}}$ \\ ${ }^{a}$ School of Economics, Centre for Behavioural and Experimental Social Science, and Centre for \\ Competition Policy, University of East Anglia, Norwich NR4 7TJ, UK \\ ${ }^{\mathrm{b}}$ Department of Economics, University of Cincinnati, Cincinnati, OH 45221, USA
}

19 September, 2016

\begin{abstract}
We analyze a group political lobbying all-pay auction with a group specific public good prize, in which one group follows a weakest-link and the other group follows a best-shot impact function. We completely characterize all semi-symmetric equilibria. There are two types of equilibria: (1) each player in the best-shot group puts mass at the upper bound of the support, whereas each player in the other group puts mass at the lower bound of the support; (2) players in the best-shot group put masses at both the lower and the upper bounds, while the other group randomizes without a mass point.
\end{abstract}

JEL Classification: C72; D70; D72; D74; H41

Keywords: group contest; all-pay auction; best-shot; weakest-link; group-specific public good

Corresponding author: Iryna Topolyan, email: topolyia@ucmail.uc.edu

* An earlier and longer version of this study was circulated under the title "The Group All-pay Auction with Heterogeneous Impact Functions". We appreciate the comments of an Associate Editor and two anonymous referees, Kyung Hwan Baik, Walter Enders, Matt Van Essen, Paan Jindapon, David Malueg, Paul Pecorino, Seth Streitmatter, Ted Turocy, the participants at the 2015 conference of 'Contest: Theory and Evidence' at the University of East Anglia, and the seminar participants at the University of Alabama and Korea University. Iryna Topolyan gratefully acknowledges the support from the Charles Phelps Taft Research Center. Any remaining errors are our own. 


\section{Introduction}

Collective rent-seeking or political lobbying can be viewed as contests in which groups collectively expend sunk resources in order to win a rent. Group members involved in rent-seeking expend resources, or 'bids,' individually. An aggregation function that maps individual group member bids into a 'group bid' is called an impact function. One of the most popular forms of modeling the winning probability is a group all-pay auction, in which only the group that expends the highest group bid wins the rent. ${ }^{1}$ In this study we focus on the specific class of rent-seeking games, in which one group is represented by the weakest bid among its group members, while the other group is represented by the strongest one.

There are various instances of such a structure in real life. Consider, for example, a lobbying game between the car industry versus the environmental groups. Car industry lobby groups, such as the Association des Constructeurs Européens d'Automobiles or Alliance of Automobile Manufacturers, often lobby for relaxed environmental regulations, car manufacturing regulations, etc., whereas environmental lobby groups, e.g., Clean Air Watch or Environmental Defense Fund, lobby for stricter rules. Oftentimes (e.g., over the past decade in California) the effective strength of the car lobby groups becomes the weakest part of the car technology, whereas the strength of the environmental groups is the strongest evidence of environmental damages. Another example comes from political economy. Imagine a status-quo policy being challenged by activists. The status-quo could be 'Britain stays in the EU' or 'Catalonia stays in Spain,' with the alternative 'Exit the status-quo.' The strength of the incumbents defending the status-quo is as good as the weakest feature of the status-quo. The strength of the challengers, however, is the best possible outcome from the alternative. Both of these examples resemble our structure. ${ }^{2}$

Note that if a group wins the contest, then it implies a win for every group member, because the prize has the feature of a group-specific public good. Hence, in this study we consider a group all-pay auction with a group specific public good prize in which one group follows a best-shot and

\footnotetext{
${ }^{1}$ Equilibria in individual all-pay auction are characterized by Baye et al. (1996), who find no pure strategy equilibrium. Players randomize over a continuous support and may put mass at some points. The upper bound of the support, the amount of the mass, and the equilibrium payoffs depend on the prize valuation.

${ }^{2}$ Further field observations consist of group conflicts in which one group defends from another group's attack. When terrorist groups attack, one can argue that the best-shot of the attack constitutes the power of terrorists, while for the internal security groups the strength of defense depends on the weakest-link; a prominent example is due to Conybeare et al. (1994). The same logic applies to the attack and defense mechanisms in computer security and in system reliability (Varian, 2004).
} 
the other group follows a weakest-link impact function. Baik et al. (2001) are the first to analyze an all-pay auction with a group specific public good prize. However, in their study, the sum of all group members' bids constitutes the group-bid. They find free-riding equilibria in which the highest valuation player in a group makes a positive bid with a positive probability, whereas all the other group-members free-ride by placing zero bids. This is later generalized by Topolyan (2014) who characterizes further equilibria. Chowdhury et al. (2016) and Barbieri et al. (2014) extend this by considering a weakest-link (the weakest or the minimum bid within a group represents the group-bid) and a best-shot (the strongest or the maximum bid does so) impact function, respectively. Both studies find multiple equilibria that can be ranked according to payoffs. ${ }^{3}$ Note that in each of those studies the impact function, i.e., the bid aggregation technology, is symmetric among the contesting groups, whereas we allow for asymmetric impact functions.

Our analysis contributes to three areas of literature. It extends the research on group contest (as it considers heterogeneous impact functions among groups) and adds to the understanding of the issues of attack and defense. Since the prize is a group-specific public good and the group members face network externalities, this study also contributes to the literature on public goods with network externalities (Hirshleifer, 1983; Bliss and Nalebuff, 1984; Bergstrom et al., 1986; Cornes, 1993; Barbieri and Malueg, 2014). Finally, the current study contributes to the all-pay auction literature by deriving new types of equilibria, in which players place mass at the upper bound of the support, or have two mass points at the opposite ends of equilibrium support.

The rest of the paper proceeds as follows. In the next section we formally set up the model. In Section 3 we solve for equilibrium and interpret the results. We provide two specific examples of the model and the corresponding equilibria in Section 4. Section 5 concludes.

\section{Model}

Consider a situation in which two groups are engaged in a group all-pay auction. Group 1 (2) consists of $m(n)$ risk-neutral players, respectively, who make irreversible and costly bids to win

\footnotetext{
${ }^{3}$ There also is a stream of research that considers group contests with the Tullock (1980) CSF. The earliest study in this area is by Katz et al. (1990) who employ an additive impact function. This is extended in various ways by Baik (1993, 2008), Lee (2012), Kolmar and Rommeswinkel (2013), Chowdhury et al. (2013) and Chowdhury and Topolyan (2016). The last study considers heterogeneous impact functions and is the closest to the current analysis. We compare these two studies below and further in the Conclusion section.
} 
a group-specific public-good prize. This bid can be in the form of money, time, effort or any other resources - depending on the specific contest, but is measured in the same units as the prize values. The individual group members' valuation for the prize may differ across groups; however it is the same within a group. Let $v_{g}>0$ represent the group-specific common valuation for the prize in group $g$. Furthermore, let $x_{g i} \geq 0$ represent the bid by player $i$ in group $g$, and $X_{g}$ - group $g$ 's bid. We assume that the group-bid of group 1 is represented by the maximum bid placed among the players in group 1, i.e., $X_{1}=\max \left\{x_{11}, x_{12}, \ldots, x_{1 m}\right\}$, while the group-bid of group 2 is determined by the minimum bid, i.e., $X_{2}=\min \left\{x_{21}, x_{22}, \ldots, x_{2 n}\right\}$. We analyze a group all-pay auction and assume that the winning probability of group 1 is

$$
p_{1}\left(X_{1}, X_{2}\right)= \begin{cases}1 & \text { if } X_{1}>X_{2} \\ 1 / 2 & \text { if } X_{1}=X_{2} \\ 0 & \text { if } X_{1}<X_{2}\end{cases}
$$

The probability that group 2 wins is: $1-p_{1}\left(X_{1}, X_{2}\right)$.

We assume that only the members of the winning group receive the group-specific public good prize but all players forgo their bids. For all the players, the cost of bid is the bid itself. Hence, the payoff for player $i$ in group $g$ is:

$$
u_{g i}=p_{g} v_{g}-x_{g i} \text {. }
$$

To close the structure, we assume that every player in the contest simultaneously and independently chooses her bid, and that all of the above, including the parameter values and the rules of the game, is common knowledge. We employ Nash equilibrium as our solution concept and use the following definitions throughout the paper. To simplify notations, Definition 1 is given for group 1, though it is analogous for group 2.

Definition 1. A strategy of player $i$ in group 1 is a probability distribution over a subset of $\mathbb{R}_{+}$, and is denoted by $s_{1 i}$. Group 1 's strategy is an $m$-tuple of its members' strategies $\left(s_{11}, \cdots, s_{1 m}\right)$, and is denoted by $s_{1}$.

Definition 2. If player $i$ in group $g$ distributes positive mass over some nonempty subset of $\mathbb{R}_{++}$, then the player is called active. Otherwise the player is called inactive. 
Definition 3. If group $g$ distributes positive mass over some nonempty subset of $\mathbb{R}_{++}$, then the group is called active. Otherwise the group is called inactive.

\section{Equilibria}

In this section we focus on and characterize (adopting the terminology by Barbieri et al., 2014) all semi-symmetric equilibrium strategies, in which all players within the same group employ the same strategy. Asymmetric equilibria may exist, where otherwise identical players are employing different strategies. However, since all players within a group are symmetric, it is natural to look at the possible semi-symmetric equilibria, which is the scope of this study.

Lemma 1. There exists no equilibrium in which both groups are inactive.

Lemma 2. There exists no pure strategy Nash equilibrium in which at least one group is active.

The latter lemma reinstates a standard result from the all-pay auction literature. It holds because overbidding by a slight margin guarantees the victory, and thus if both groups play pure strategies (delivering a nonnegative payoff to each player in equilibrium), a player in the best-shot group would deviate and slightly increase her bid.

Next we characterize equilibrium supports. For each group $g$, let $S_{g+}^{*}$ denote $S_{g}^{*} \cap \mathbb{R}_{++}=$ $\left\{x>0: x \in S_{g}^{*}\right\}$, where $S_{g}^{*}$ represents group $g$ 's equilibrium support. Also, let $\underline{s}_{g}^{*}=\inf \left\{x: x \in S_{g}^{*}\right\}$, $\underline{s}_{g+}^{*}=\inf \left\{x: x \in S_{g+}^{*}\right\}$, and $\bar{s}_{g}^{*}=\sup \left\{x: x \in S_{g}^{*}\right\}$.

Lemma 3. Let $s^{*}=\left\{s_{1}^{*}, s_{2}^{*}\right\}$ be a mixed strategy Nash equilibrium. Then the following statements are true. $^{4}$

i. $S_{1+}^{*}=S_{2+}^{*}$.

ii. $\min \left\{\underline{s}_{1}^{*}, \underline{s}_{2}^{*}\right\}=0$.

iii. "No gaps in the support": i.e., there exists no interval $(a, b) \subseteq S_{g}^{*}$, where $a<b$, such that $F_{g}(a)=F_{g}(b), g=1,2$.

iv. $\quad$ No player in group 2 puts mass at $\overline{\mathrm{s}}^{*}$.

\footnotetext{
${ }^{4}$ The proofs are standard and omitted here; they are available upon request from the corresponding author, or can be found in the working paper version of the study in Chowdhury and Topolyan (2015).
} 
v. No group $g$ puts mass at $x$ for any $x \in\left(\underline{s}_{+}^{*}, \bar{s}^{*}\right)$.

vi. At most one group puts mass at zero.

Property (i) is inherited from the weakest-link all-pay auction, as group 2 follows the weakest-link; the proof goes along the lines of Lemma 4.1.2 in Chowdhury et al. (2016). Parts (ii), (iii), (v), and (vi) are common to the best-shot as well as the weakest-link all-pay auctions; for details, refer to Barbieri et al. (2014) and Chowdhury et al. (2016). Property (iv) is inherited from the best-shot group all-pay auction, see Barbieri et al. (2014).

Combining Lemmas 1-3, we state the main result of this analysis. ${ }^{5}$ A remarkable feature of the equilibria is that players in the best-shot group can put atom at the upper bound of the support. This is only possible because of the group-bid technology within the weakest-link group. In fact, it is possible that a group puts mass at both the lower and the upper bound of the support. This result is reminiscent of Che and Gale (1998) but it is obtained without assuming a cap on the bids.

Theorem 1. There exists a continuum of semi-symmetric equilibria of the max-min all-pay auction, characterized as follows.

1) If $\frac{n v_{1}}{m v_{2}} \geq 1$, then to every $0 \leq a_{1}<1$ there corresponds a unique equilibrium, such that every player in group 1 puts a mass of $a_{1}$ at $\bar{s}^{*}$, where $\bar{s}^{*}$ is a function of $a_{1}$, and every player in group 2 puts a mass of $\left[1-\frac{m v_{2}}{n v_{1}}\left(1-a_{1}\right)\right]$ at zero. Player 1's cdf is given by the unique solution to

$$
\frac{\left(m v_{2}\right)^{n}}{\left(n v_{1}\right)^{n-1}}(F(x))^{m-1}\left(1-F(x)-a_{1}\right)^{n-1} F^{\prime}(x)=1, F(0)=0,
$$

and player 2's cdf is given by $G(x)=1-\frac{m v_{2}}{n v_{1}}\left(1-a_{1}\right)+\frac{m v_{2}}{n v_{1}} F(x)$ for $0 \leq x \leq \bar{s}^{*}$.

2) If $\frac{n v_{1}}{m v_{2}}<1$, then there exist two types of equilibria.

I. To every $1-\frac{n v_{1}}{m v_{2}} \leq a_{1}<1$ there corresponds a unique equilibrium, such that every player in group 1 puts a mass of $a_{1}$ at $\bar{s}^{*}$, where $\bar{s}^{*}$ is a function of $a_{1}$, and every player in group 2 puts a mass of $\left[1-\frac{m v_{2}}{n v_{1}}\left(1-a_{1}\right)\right]$ at zero. Player 1's cdf is given by the unique solution to

\footnotetext{
${ }^{5}$ Furthermore, for details of further results, discussions and diagrammatic representations, please see the working paper version of this study in Chowdhury and Topolyan (2015).
} 


$$
\frac{\left(m v_{2}\right)^{n}}{\left(n v_{1}\right)^{n-1}}(F(x))^{m-1}\left(1-F(x)-a_{1}\right)^{n-1} F^{\prime}(x)=1, F(0)=0,
$$

and player 2's cdf is given by $G(x)=1-\frac{m v_{2}}{n v_{1}}\left(1-a_{1}\right)+\frac{m v_{2}}{n v_{1}} F(x)$ for $0 \leq x \leq \bar{s}^{*}$

II. To every $0 \leq a_{1} \leq 1-\frac{n v_{1}}{m v_{2}}$, there corresponds a unique equilibrium, such that every player in group 1 puts a mass of $a_{1}$ at $\bar{s}^{*}$, where $\bar{s}^{*}$ is a function of $a_{1}$, Every player in group 1 puts a mass of $\left[1-a_{1}-\frac{n v_{1}}{m v_{2}}\right]$ at zero and a mass of $a_{1}$ at $\bar{s}^{*}$. Player 2 's $c$ df is given by the unique solution to

$$
n v_{1}\left(1-a_{1}-\frac{n v_{1}}{m v_{2}}+\frac{n v_{1}}{m v_{2}} G(x)\right)^{m-1}(1-G(x))^{n-1} G^{\prime}(x)=1, G(0)=0,
$$

and player 1's cdf is given by $F(x)=1-a_{1}-\frac{n v_{1}}{m v_{1}}+\frac{n v_{1}}{m v_{2}} G(x)$ for $0 \leq x \leq \bar{s}^{*}$

Proof: See Appendix.

The remarkable multiplicity of equilibria reflects varying degrees of free-riding. Note that although every player is active, free-riding takes the form of an insufficient effort, where lower effort levels are chosen with higher probability.

Equilibria where the best-shot group puts mass at the upper bound while the weakest-link group puts mass at zero (cases 1 and 2.I), have the following property. As the size of the weakestlink group, $n$, increases (or the size of the best-shot group, $m$, decreases), each player in group 2 puts greater mass at zero, which improves the payoffs in group $1 .^{6}$ Players in group 2 always receive zero payoff as long as they put positive mass at zero. Thus, their payoff is unaffected by the changes in the group sizes, as long as $n v_{1} \geq m v_{2}$ is satisfied, although the players bid less aggressively. On the other hand, when players in group 1 put masses at zero as well as the upper bound (case 2.II), every player in group 2 receives a positive expected payoff of $\left(1-a_{1}-\frac{n v_{1}}{m v_{2}}\right)$, which diminishes as $n$ increases (or $m$ decreases), as long as $\frac{n v_{1}}{m v_{2}}<1$. $^{7}$

We, therefore, find partial support of the 'group size paradox' (Olson, 1965; Bergstrom et al., 1986; Pecorino, 2015); the inverse relation between the group size and payoffs in that group

\footnotetext{
${ }^{6}$ Observe that the mass that each player in group 2 puts at zero is inversely related to $a_{1}$. The upper bound of the support, $\bar{s}^{*}$, is determined from equation $A_{1}\left(1-a_{1}\right)=\bar{s}^{*}$, where $A_{1}$ is strictly increasing in the relevant range. Thus when every player in group 2 puts greater mass at zero, $\bar{s}^{*}$ decreases, making players in group 1 better-off.

${ }^{7}$ In general it is difficult to say how the upper bound of the group support varies with the group sizes, as the expression in (7) is complicated. Hence it is difficult to say how players in group 1 fare as the group sizes change.
} 
holds under certain conditions, while there may be no relation at all (a constant payoff of zero), or the relation may be ambiguous.

\section{Examples}

In the previous section we characterized the equilibria of the game. We observed that two cases may arise that are very different qualitatively. Here we provide with examples that delineate the two most basic types of the equilibria, but cover both the technicalities and the intuition regarding symmetry vs. asymmetry in the group size and in prize valuations. The first one considers symmetric groups with common prize value whereas the second one incorporates different group sizes with different prize values across groups. These also correspond to the two types of the equilibria characterized.

\subsection{Example 1: the symmetric group size, symmetric value case}

Suppose the groups are symmetric, with $m=n=2$ and $v_{1}=v_{2}=1$. Theorem 1 implies there exists a continuum of equilibria, in which each player in group 1 puts a mass of $a_{1}$ at the upper bound of the common support, where $0 \leq a_{1}<1$, and every player in group 2 puts the same mass at zero. Equation (5) implies:

$2 F(x)\left(1-F(x)-a_{1}\right) F^{\prime}(x)=1$.

Let $y=F(x)$, then the following equation implicitly defines $y$ as a function of $x$ under the condition $0 \leq y \leq 1-a_{1}$ :

$y^{2}\left(1-a_{1}\right)-\frac{2}{3} y^{3}=x$.

The implicit plot of (9) for the case $a_{1}=0$ is in Figure 4. Indeed, when we restrict our attention to the range $\{y: 0 \leq y \leq 1\}, y$ is implicitly defined as an increasing function of $x$. 


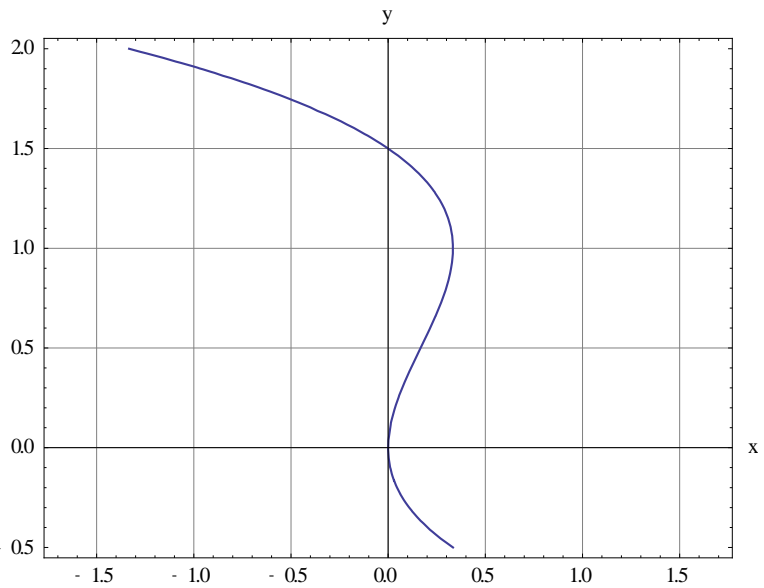

Figure 4. Implicit plot of $y^{2}-\frac{2}{3} y^{3}=x$.

The family of CDFs of a player in group 1 , when $a_{1}$ takes values of $0,0.1,0.2$, and 0.5 , is in Figure 5. Here effort levels are depicted on the horizontal axis, and values that CDF takes are depicted on the vertical axis. Theorem 1 implies that every player in group 2 puts the same mass at zero. It is easy to see that the upper bound of the groups' support is decreasing in $a_{1}$ and ranges from 0.33 when $a_{1}=0$ to 0.04 when $a_{1}=0.5$. The upper bound of the support goes to zero as $a_{1}$ goes to 1 . Notice that in equilibrium $a_{1}=1$ is never achieved (since no group sustain a pure strategy), suggesting that the NE correspondence is not upper hemicontinuous. This happens because the players' payoffs are discontinuous due to the all-pay auction assumption.

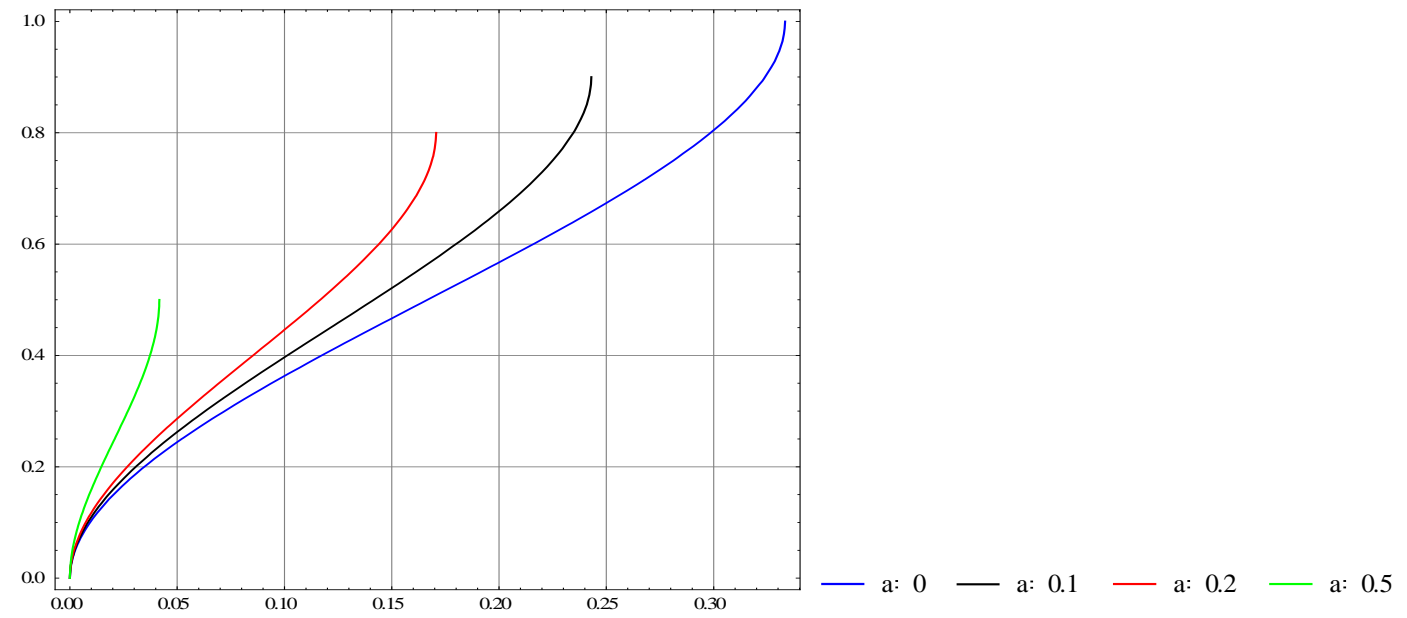

Figure 5. Strategy (CDF) of a player in group 1 when $a_{1}=0,0.1,0.2$, and 0.5 . 


\subsection{Example 2: the asymmetric group size, asymmetric value case}

Now suppose group 1 has three players with valuation $v_{1}=1$, and group 2 has two players with valuation $v_{2}=3$. There are two types of equilibria.

Type I equilibria: each player in group 1 puts a mass of $a_{1}$ at the upper bound of the common support, where $1 / 2 \leq a_{1}<1$, and every player in group 2 puts a mass of $a_{G}=\frac{1}{2}(1+$ $\left.a_{1}\right)$ at zero. Equation (5) implies

$24 F(x)\left(1-F(x)-a_{1}\right)^{2} F^{\prime}(x)=1$.

Letting $y=F(x)$, we obtain the following equation relating $y$ and $x$, where $0 \leq y \leq 1-a_{1}$ :

$6 y^{4}+16\left(a_{1}-1\right) y^{3}+12\left(a_{1}-1\right)^{2} y^{2}=x$.

CDFs of a player in group 1 , when $a_{1}$ takes values of $0.5,0.6$, and 0.7 , are depicted in Figure 6. Again, the upper bound of the groups' support diminishes from 0.125 to zero as $a_{1}$ increases. As $a_{1}$ increases, players in group 2 put larger mass at zero, ranging from 0.75 to 1 . As before, the mass gets infinitely close to one, however the maximum of one is never achieved.

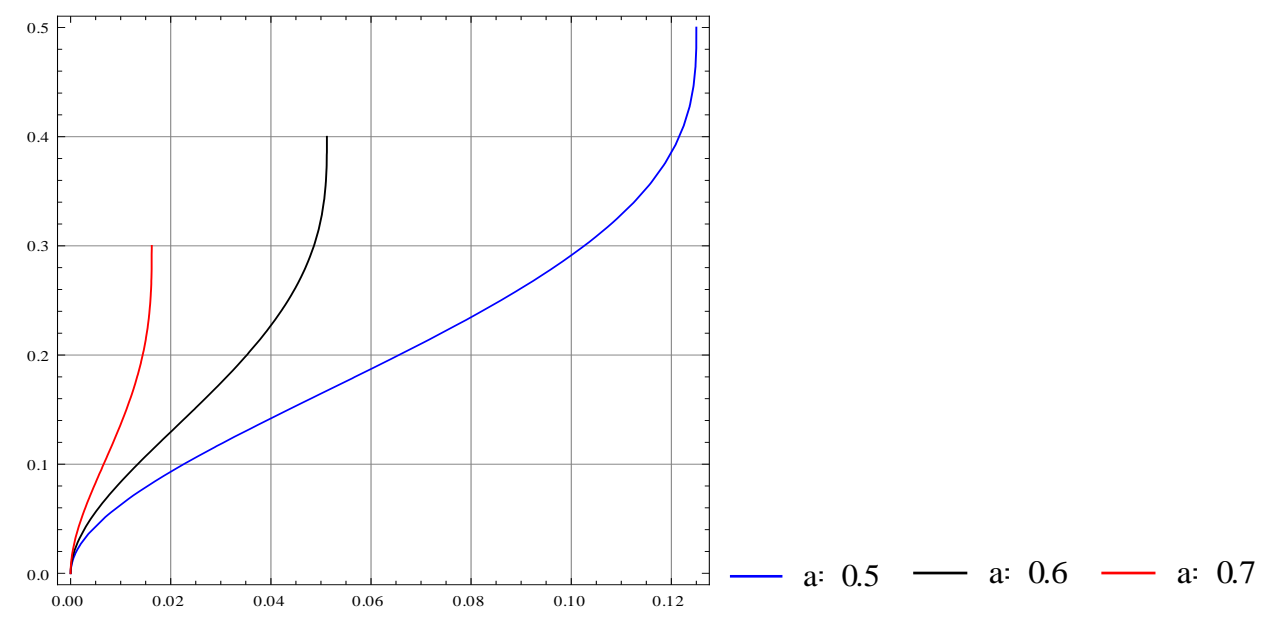

Figure 6. Strategy (CDF) of a player in group 1 when $a_{1}=0.5,0.6$, and 0.7 .

Type II equilibria: each player in group 1 puts a mass of $a_{1}$ at the upper bound of the common support, where $0 \leq a_{1} \leq \frac{1}{2}$, and a mass of $a_{F}=\frac{1}{2}-a_{1}$ at zero. Equilibrium CDFs of a player in group 2 when $a_{1}$ takes values of $0,0.25$, and 0.5 are depicted in Figure 7. 


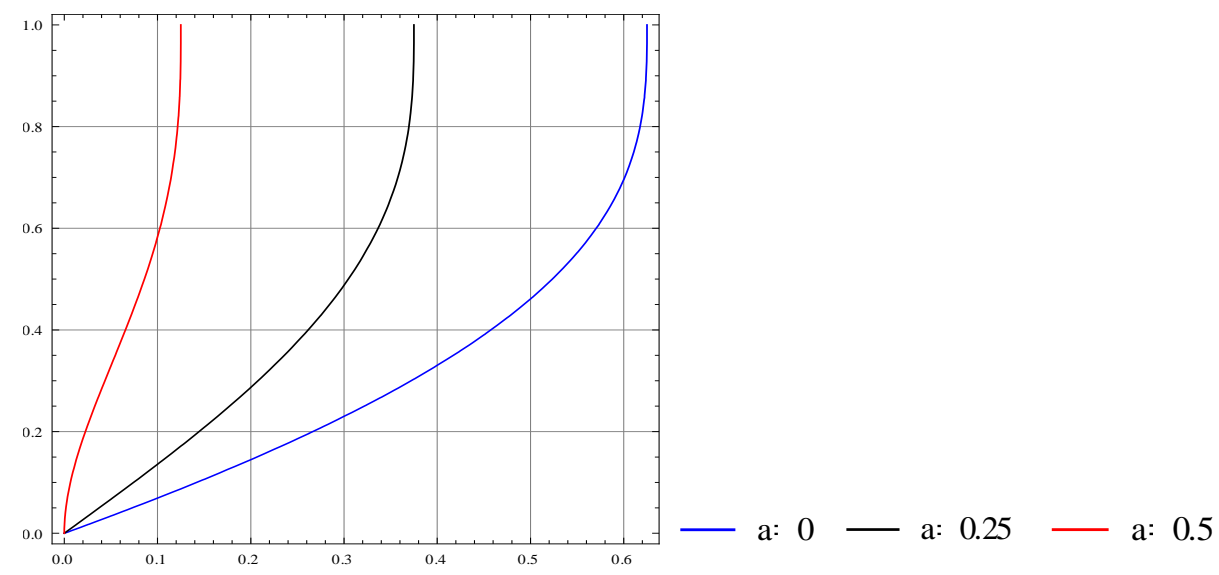

Figure 7. Strategy (CDF) of a player in group 2 when $a_{1}=0,0.25$, and 0.5 .

As can be observed from the figure above, the upper bound of the support diminishes, similar to the last case, as $a_{1}$ increases.

\section{Conclusion}

We introduce a group rent-seeking contest with all-pay auction CSF and heterogeneous impact functions. Compared to the existing studies of group all-pay auctions, we find equilibria that are qualitatively different. While Chowdhury et al. (2016), imposing weakest-link impact functions for both groups, find equilibria in which pure and mixed strategies may co-exist, only mixed strategies could be sustained in equilibrium in our model. Also, unlike Chowdhury et al. (2016), it is not possible for both groups to be inactive in equilibrium. These results stem from the fact that one group adopts the best-shot effort technology. As expected, our results are also very different from those of Baik et al. (2001), who employ perfect substitute impact functions for both groups and find free-riding equilibria that are similar to the standard all-pay auction equilibria of Baye et al. (1996). Chowdhury and Topolyan (2016) analyze group contest with heterogeneous impact functions but with logit-type (Tullock, 1980) CSF. They characterize pure strategy equilibria and show that in any equilibrium only one player in the attacker group is active, while all players in the defender group exert the same positive effort. We show, compared to Chowdhury and Topolyan (2016), that much wider participation is possible in mixed strategy equilibria. Particularly, we show that it is possible that all members in the best-shot group are active and randomize over the 
same support, and that members of the weakest-link group may fail to coordinate with respect to the effort level and exert different efforts in a mixed strategy equilibrium.

We provide an important step to a flourishing area of literature. There are various ways to broaden this analysis, which are out of scope of the current study. We consider the case where the moves are simultaneous. It is, however, easy to show that the equilibria still prevail in a situation where the moves are sequential. Since the best responses remain the same, the result will also be the same when only one move per group is considered. However, the results will be different when the groups are allowed to add to the existing bids in a sequence. A technically difficult extension would be to allow more than two groups and to introduce heterogeneous valuations. Endogenizing the choice of impact function, endogenizing group formation, considering private good prizes, are among further possible topics. It will also be possible to consider simultaneous inter- and intragroup conflict (e.g., Choi et al., 2016) in this asymmetric impact function setting. Finally, we consider a complete information structure; introducing incomplete information may provide interesting results.

\section{References}

Baik, K.H. (1993). Effort levels in contests: The public-good prize case. Economics Letters, 41(4), 363-367.

Baik, K.H. (2008). Contests with group-specific public-good prizes. Social Choice and Welfare, 30(1), 103-117.

Baik, K.H., Kim, I.G., \& Na, S. (2001). Bidding for a group-specific public-good prize. Journal of Public Economics, 82(3), 415-429.

Barbieri, S., \& Malueg, D.A. (2014). Group efforts when performance is determined by the "best shot". Economic Theory, 56(2), 333-373.

Barbieri, S., Malueg, D.A., \& Topolyan, I. (2014). The best-shot all-pay (group) auction with complete information. Economic Theory, 57(3), 603-640.

Baye, M. R., Kovenock, D., \& De Vries, C.G. (1996). The all-pay auction with complete information. Economic Theory, 8(2), 291-305.

Bergstrom, T., Blume, L., \& Varian, H. (1986). On the private provision of public goods. Journal of Public Economics, 29(1), 25-49. 
Bliss, C., \& Nalebuff, B. (1984). Dragon-slaying and ballroom dancing: The private supply of a public good. Journal of Public Economics, 25(1), 1-12.

Che, Y.-K., Gale, I. (1998). Caps on Political Lobbying, American Economic Review, 88(3), 643651.

Choi, J.P., Chowdhury, S.M., \& Kim, J. (2016). Group Contests with Internal Conflict and Power Asymmetry, Scandinavian Journal of Economics, Forthcoming.

Chowdhury, S.M., Lee, D., \& Sheremeta, R.M. (2013). Top guns may not fire: Best-shot group contests with group-specific public good prizes. Journal of Economic Behavior \& Organization, 92, 94-103.

Chowdhury, S. M., Lee, D., \& Topolyan, I. (2016). The Max-Min Group Contest: Weakest-link (Group) All-Pay Auction. Southern Economic Journal, Forthcoming.

Chowdhury, S. M., \& Topolyan, I. (2015). The Group All-Pay Auction with Heterogeneous Impact Functions. University of East Anglia Economics Working paper No. 069.

Chowdhury, S. M., \& Topolyan, I. (2016). The Attack-And-Defense Group Contests: Best Shot Versus Weakest Link. Economic Inquiry, 54(1), 548-557

Conybeare, J.A., Murdoch, J.C., \& Sandler, T. (1994). Alternative collective-goods models of military alliances: Theory and empirics. Economic Inquiry, 32(4), 525-542.

Cornes, R. (1993). Dyke maintenance and other stories: Some neglected types of public goods. The Quarterly Journal of Economics, 259-271.

Hirshleifer, J. (1983). From weakest-link to best-shot: The voluntary provision of public goods. Public Choice, 41, 371-386.

Katz, E., Nitzan, S., \& Rosenberg, J. (1990). Rent-seeking for pure public goods. Public Choice, 65(1), 49-60.

Kolmar, M., \& Rommeswinkel, H. (2013). Contests with group-specific public goods and complementarities in efforts. Journal of Economic Behavior \& Organization, 89, 9-22.

Lee, D. (2012). Weakest-link contests with group-specific public good prizes. European Journal of Political Economy, 28(2), 238-248.

Olson, M. (1965). The Logic of Collective Action: Public Goods and the Theory of Groups. Harvard University Press, Cambridge, MA.

Pecorino, P. (2015). Olson's logic of collective action at fifty. Public Choice, 162, 243-262. 
Topolyan, I. (2014). Rent-seeking for a public good with additive contributions. Social Choice and Welfare, 42(2), 465-476.

Tullock, G. (1980). Efficient Rent Seeking. In James M. Buchanan, Robert D. Tollison, Gordon Tullock, (Eds.), Toward a theory of the rent-seeking society. College Station, TX: Texas A\&M University Press, 97-112.

Varian, H.A. (2004). System reliability and free riding, Economics of Information Security. Advances in Information Security, 12, 1-15. 


\section{APPENDIX}

\section{Proof of Theorem 1.}

Let each player in group 1 randomize according to some CDF $F(\cdot)$, and each player of group 2 randomize according to a CDF $G(\cdot)$. Following Barbieri et al. (2014), the expected payoff of player $i$ in group 1 from exerting an effort of $x$ is given by

$$
u_{1 i}(x)=-x+v_{1}\left[1-\int_{x}^{\infty}(F(z))^{m-1} n(1-G(z))^{n-1} d G(z)\right] .
$$

Then, $u_{1 i}^{\prime}(x)=n v_{1}(F(x))^{m-1}(1-G(x))^{n-1} G^{\prime}(x)-1=0$, which implies

$n v_{1}(F(x))^{m-1}(1-G(x))^{n-1} G^{\prime}(x)=1$.

Analogously,

$m v_{2}(F(x))^{m-1}(1-G(x))^{n-1} F^{\prime}(x)=1$.

Let $a_{1}$ be the (possibly zero) mass that every player in group 1 puts at $\bar{s}^{*}$, then dividing (2) by (3) and integrating over $\left[x, \bar{s}^{*}\right]$ yields

$1-G(x)=\frac{m v_{2}}{n v_{1}}\left(1-F(x)-a_{1}\right)$ for all $0<x<\bar{s}^{*}$.

Consider two possibilities.

Case I. Each player in group 2 puts a mass of $a_{G}$ at zero, where $0 \leq a_{G}<1$. Lemma 3(vi) implies that players in group 1 do not put mass at zero. Equation (4) could be written as $G(x)=1-$ $\frac{m v_{2}}{n v_{1}}\left(1-a_{1}\right)+\frac{m v_{2}}{n v_{1}} F(x)$, therefore in order to satisfy $0 \leq a_{G}<1$, we must have $a_{1} \geq 1-\frac{n v_{1}}{m v_{2}}$. Notice that if $\frac{n v_{1}}{m v_{2}} \geq 1$, this condition holds for all $0<a_{1}<1$.

Plugging (4) into (3) yields

$\frac{\left(m v_{2}\right)^{n}}{\left(n v_{1}\right)^{n-1}}(F(x))^{m-1}\left(1-F(x)-a_{1}\right)^{n-1} F^{\prime}(x)=1$.

Denote $\frac{\left(m v_{2}\right)^{n}}{\left(n v_{1}\right)^{n-1}} y^{m-1}\left(1-y-a_{1}\right)^{n-1}$ by $D_{1}(y)$. Note that since $D_{1}(y)$ is a polynomial, its anti-derivative exists and is also a polynomial. Denote by $A_{1}(y)$ the antiderivative of $D_{1}(y)$ 
with zero constant term. The solution to (5) has the form $A_{1}[F(x)]=x+C$, where $C=$ constant. Since by assumption $F(0)=0$, the constant $C$ is equal to zero. Thus a solution to (5) satisfies $A_{1}[F(x)]=x$.

Furthermore, $D_{1}[F(x)] \geq 0$ if and only if $0 \leq F(x) \leq 1-a_{1}$, with strict inequality whenever $F(x)$ is in the interior of the interval. Notice that since $D_{1}(y)=\frac{\partial}{\partial y}\left[A_{1}(y)\right], A_{1}(\cdot)$ is strictly increasing on $\left(0,1-a_{1}\right)$. Therefore the inverse function $A_{1}^{-1}$ is defined for all $0<F(x)<$ $1-a_{1}$, and thus there exists a unique solution to (5) satisfying $0<F(x)<1-a_{1}$. The upper bound of the support $\bar{s}^{*}$ is determined uniquely from equation $A_{1}\left(1-a_{1}\right)=\bar{s}^{*}$, because gaps in the support of $F$ are ruled out by Lemma 3(iii). Notice that by assumption we have $F(0)=0$ and $F\left(\bar{s}^{*}\right)=1-a_{1}$.

Finally, observe that the mass point at the upper bound of the support does not provide profitable deviations in either group. Indeed, a deviation to a lower effort level is not profitable for group 1 (group 2) by Equation 2 (Equation 3), while an upwards deviation for group 1 is not profitable because the other group does not put mass at the upper bound. An upwards deviation for group 2 is not profitable because of the weakest-link effort technology in this group.

Case II. Each player in group 1 puts a mass of $a_{F}$ at zero, where $0 \leq a_{F}<1$, while players in group 2 do not put mass at zero. Rewrite Equation (4) as $F(x)=1-a_{1}-\frac{n v_{1}}{m v_{1}}+\frac{n v_{1}}{m v_{2}} G(x)$, then in order to satisfy $0 \leq a_{F}<1$, we must have $a_{1} \leq 1-\frac{n v_{1}}{m v_{2}}$, which holds only if $\frac{n v_{1}}{m v_{2}}<1$. The latter condition is satisfied for all $0<a_{1}<1$ when $\frac{n v_{1}}{m v_{2}} \geq 1$. Similarly to the previous case,

$n v_{1}\left(1-a_{1}-\frac{n v_{1}}{m v_{2}}+\frac{n v_{1}}{m v_{2}} G(x)\right)^{m-1}(1-G(x))^{n-1} G^{\prime}(x)=1$

Letting $D_{2}(y)=n v_{1}\left(1-a_{1}-\frac{n v_{1}}{m v_{2}}+\frac{n v_{1}}{m v_{2}} y\right)^{m-1}(1-y)^{n-1}$ and denoting by $A_{2}(y)$ the antiderivative of $D_{2}(y)$ with zero constant term, and then following the lines of Case I and noting that $A_{2}$ is strictly increasing on $(0,1)$ conclude that there exists a unique solution to $(7)$. Finally, from $A_{2}(1)=\bar{s}^{*}$ we solve for $\bar{s}^{*}$. To complete the proof, follow Case I to conclude that a mass point at the upper bound of the support does not allow profitable deviations. 